\title{
New Neutron Imaging Facility development at the Penn State Breazeale Nuclear Reactor
}

\author{
Alibek Kenges, Kenan Unlu, Daniel Beck \\ Pennsylvania State University, USA \\ Corresponding author: azk63@psu.edu
}

\begin{abstract}
Preliminary results of characterization experiments for the New Neutron Imaging Facility (NIF) that is being developed at the Penn State Breazeale Nuclear reactor are presented in the following sections. The methodology of neutron beam characterization described in the American Society for Testing and Materials (ASTM) documents for the neutron imaging systems have been followed to improve the NIF at Penn State to a Category I facility by ASTM designation of quality. Preliminary results showed that our system is capable of producing images of high quality, corresponding to Category I; however, further experiments are needed for full declaration of our facility as such. Additionally, the effective collimation ratio (L/D ratio) of our current system is $\sim 110$ with the capability of improvement to $\sim 150$. The thermal flux at the exit surface of the neutron beam is equal to $5.4 \times 10^{6} \mathrm{ncm}^{-2} \mathrm{~s}^{-1}$ at $1 \mathrm{MWth}$ reactor power, which corresponds to the industry comparable value.
\end{abstract}

Keywords - Neutron imaging, neutron radiography, neutron filters, collimator components, beam characterization.

\section{INTRODUCTION}

$\mathrm{N}$ EUTRON imaging can be described as one of the most useful techniques in non-destructive testing field that effectively uses the attenuation properties of neutrons to image the objects of interest. A neutron imaging facility has been available at Radiation Science and Engineering Center (RSEC) for the last several decades and with the newly installed core moderator assembly and the beam ports, it has been decided to rejuvenate the NIF at RSEC to its new levels. The new NIF has collimators installed over the past several months with the capability of utilizing variable apertures. It is also on the right track of using the state-of-the-art equipment and software for conventional neutron radiography and tomography. The potential of all RSEC facilities is highlighted by the Penn State Breazeale Reactor (PSBR), which first went critical in 1955 , and is the nation's longest continuously operating university research reactor. The PSBR is a $1 \mathrm{MW}$, TRIGA with moveable core in a large pool and with pulsing capabilities [1]. The current layout for the RSEC NIF and the beam port dimensions are given in the Figure 1. Although the final design for the RSEC NIF is still under development, it has been decided to continuously characterize our system by the universal standards for the Neutron Radiography (NR). This would allow us to properly examine the capabilities of the facility at each point of the development process. While there are some other ways, most of the NR facilities around the world characterize their systems using American Society for Testing and Materials (ASTM) standards dedicated to neutron imaging techniques.

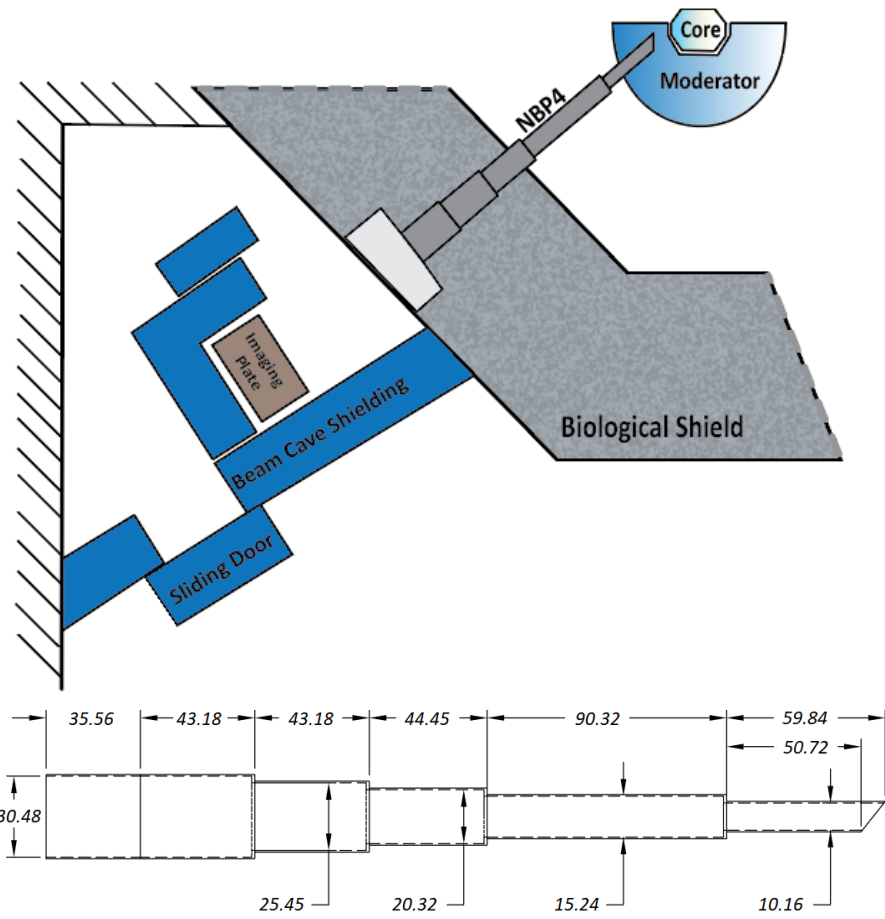

Fig. 1. RSEC NIF layout and the beam port (dimensions are in centimeters).

Therefore, it has been decided to follow the same methodology in categorization process of RSEC - NIF. The starting point of the system characterization was the assessment of Open Beam (OB) configuration, which is the beam port that does not have any collimator components installed [2]. Once the collimators components are installed the ASTM testing can be repeated to see the improvements in the system, and those steps are briefly summarized in the following section.

\section{CHARACTERIZATION OF THE RSEC - NIF}

Although we have the capability of implementing the film radiography, for these characterizations indirect method has been utilized, which assumes the conversion plate to be exposed without the film [3]. Instead, our system exploits the computed 
radiography (CR) technique that is made possible by the CRxFlex by Baker Hughes GE company and corresponding IPU phosphor imaging plates.

The OB configuration of the RSEC - NIF has been characterized by the end of the 2020. As a result, it has been determined that the images of medium level of quality can be produced corresponding to a facility of Category IV by ASTM designation of quality. The objective then was to improve the system in all NR relevant aspects to achieve the Category I via installation of collimator components shown in the figures below and discussed further.

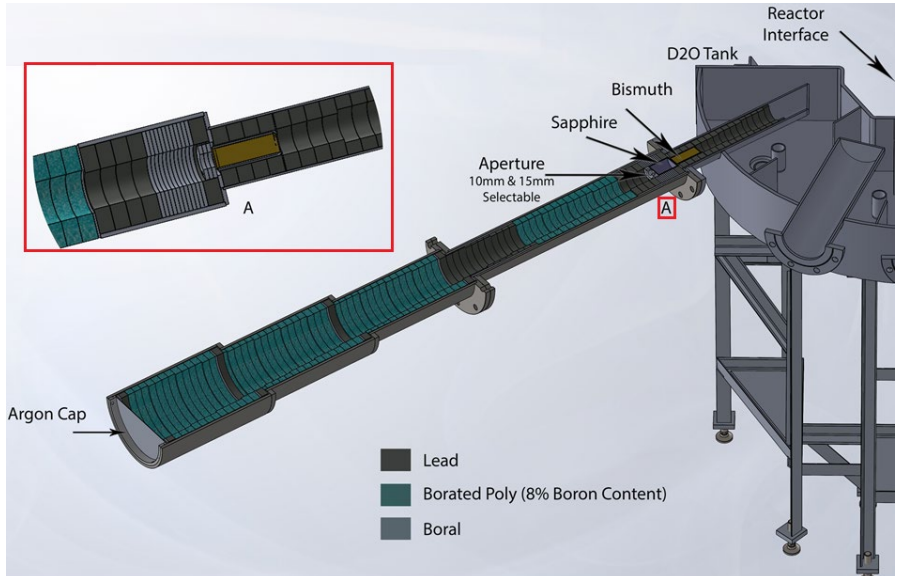

Fig. 2. Collimator components of the RSEC - NIF beam port. Note: Both, the Bismuth and Sapphire filters can be removed and placed to the beam port depending on the neutron spectrum needs.

To improve the collimation ratio, it has been decided to manufacture the aperture with the selectable diameters. Boral apertures of $20 \mathrm{~mm}$ and $30 \mathrm{~mm}$ in diameter have been selected as the preliminary suitable choice. There is the upper limit for the aperture diameter due to the diameter of the Bi-filter, which is $36 \mathrm{~mm}$; however, the lower limit is only dependent on the value of the desired thermal neutron flux that will be reduced with the smaller diameter apertures. Optionally, the cadmium lining can also be added to the aperture to sharpen the edges.

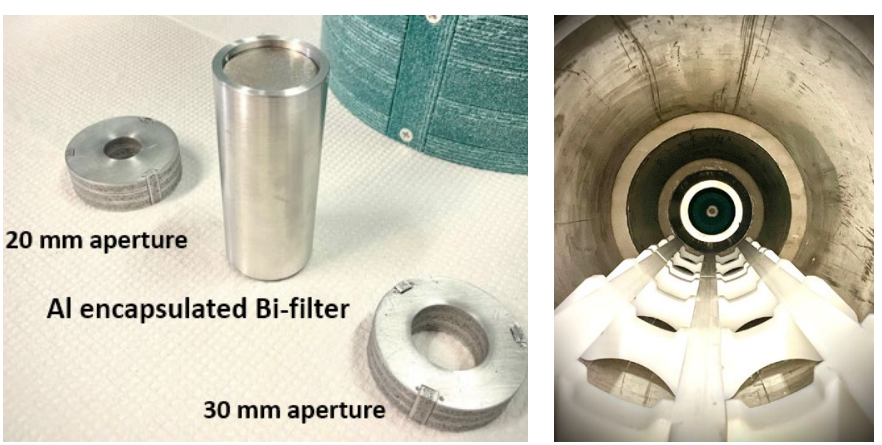

Fig.3. Collimator components for the RSEC - NIF beam port: Bi single crystal in the aluminum encapsulation and two boral apertures (left), installation of the lead and poly steps using the in-house 3D printed delivery rails (right).

As the collimator beam walls (convergent and divergent parts), the lead and borated-poly material ( $8 \%$ Boron content) steps have been chosen with the filters for the gammas (Bismuth) and optional filter for the fast neutrons (Sapphire) can be installed pre and post aperture respectively. The choice for the mentioned collimator components were based on analysis of the existing NR facilities and runs of the appropriate MCNP calculations.
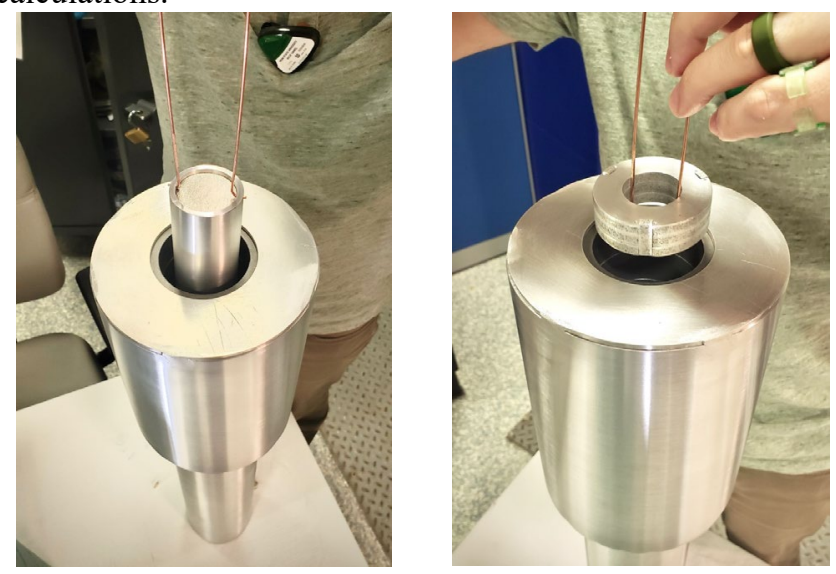

Fig.4. Collimator components for the RSEC - NIF beam port: insertion of the Bi-gamma-filter (left) and the boral aperture (right) in the corresponding section of the beam port.

The figure above shows of the installation process of the collimator components. It should be noted that this process can also be performed in the horizontal position once the components are installed in the beam port. The resulting view of the Collimated Beam (CB) port of the RSEC - NIF with all collimator components installed is shown in the figure below.
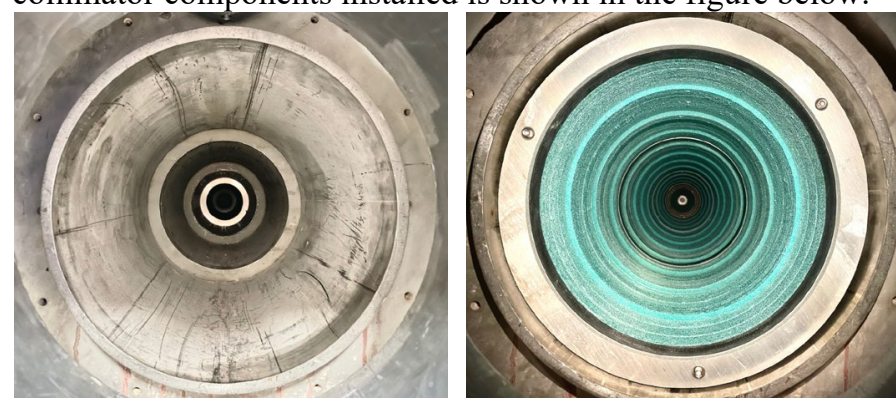

Fig.5. The RSEC - NIF beam port (view towards the reactor); OB (left) and CB (right).

The assessment of the system can be started by the examining the uniformity of the beam, which can be done by taking the image without any object placed within the beam. To consider the beam as uniform, the optical density values across the resulting radiograph should not deviate more than $5 \%$ as indicated in ASTM E545 [3]. In the figure below, although the intensities of the grey values for the $\mathrm{OB}$ and $\mathrm{CB}$ configurations are slightly differ, better uniformity in the beam profile can be seen for the latter.

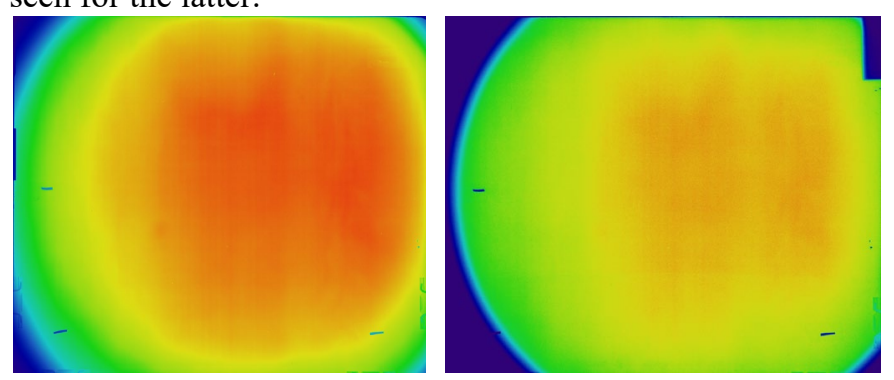

Fig. 6. The resulting image of the beam profile for the $\mathrm{OB}$ (left) and $\mathrm{CB}$ (right) configurations (a filter is used for visual illustration). 
The difference in the grey value intensity is first due to the reduction in the thermal neutron flux at the surface of imaging plate. Gold foil activation technique was used for the experimental values of thermal neutron flux, that resulted in $3.7 \times 10^{8} \mathrm{ncm}^{-2} \mathrm{~s}^{-1}$ per $1 \mathrm{MW}$ reactor power for the OB configuration. On the other hand, the value was reduced to $5.4 \times 10^{6} \mathrm{ncm}^{-2} \mathrm{~s}^{-1}$ per $1 \mathrm{MW}$ power for the $\mathrm{CB}$ configuration. Deviation of the grey values across the entire image in Fig. 3 changed from $\sim 40 \%$ for the OB to $\sim 24 \%$ for the $\mathrm{CB}$. In the middle section of the image these numbers changed from $\sim 13 \%$ to $\sim 10 \%$. Both values are more than $5 \%$ to declare the beam as uniform; however, this issue could be related to IPU phosphor imaging plate and its condition throughout the experiments. To make it right, the direct film radiography needs to be performed to access the beam uniformity for the $\mathrm{CB}$ configuration.

\section{A. Effective Collimation Ratio (L/D)}

Collimation ratio (L/D) of the imaging system defines the spatial resolution and hence can be treated as one of the most important features. The length of the beam port from the aperture the beam shutter end surface would be equal to 257 $\mathrm{cm}$. Therefore, depending on the placement of the imaging plate post beam shutter, the value of $(\mathrm{L})$ can be anywhere between 260 to around 600 . On the other hand, the diameter of the aperture (D) is currently selectable between $20 \mathrm{~cm}$ and $30 \mathrm{~cm}$. Simple division of two values would result in the geometric L/D but the effective collimation ratio, on the other hand, differs from the geometrical ratio due to the scattering events within the beam. ASTM E803 document provides the details of the experiment to find the effective $\mathrm{L} / \mathrm{D}$ of the system using the no umbra (NU) device [4]. Two resulting images for this device are given in the figure below for the $\mathrm{OB}$ and $\mathrm{CB}$ configurations.
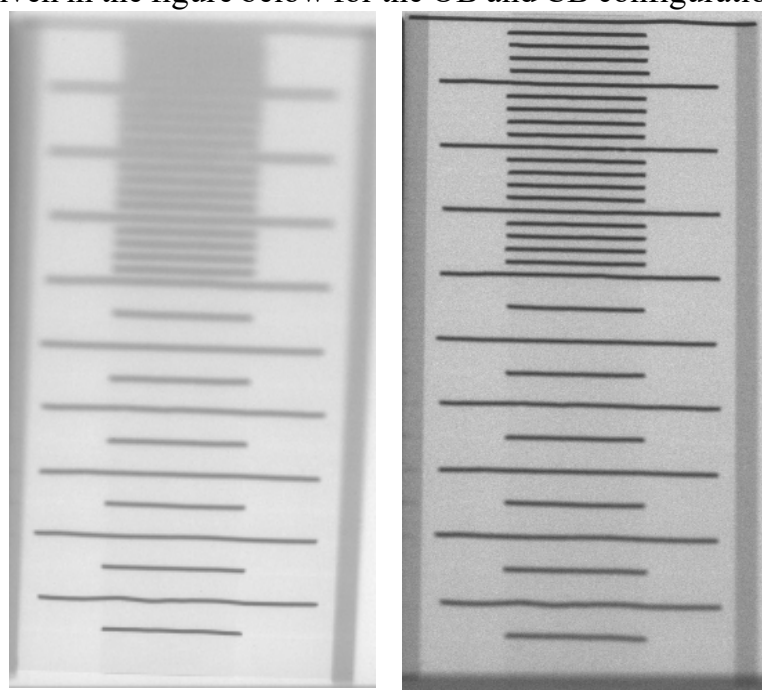

Fig. 7. The resulting image of the NU-device for the $\mathrm{OB}$ (left) and $\mathrm{CB}$ (right) configurations. Note: In general, sharp shadow lines (umbra shadow) of Cdwires represent higher $\mathrm{L} / \mathrm{D}$ value.

There are two different techniques of finding the effective $\mathrm{L} / \mathrm{D}$ value by analyzing the image of NU-device that are given in ASTM E803 document. One of them is the visual analysis of the NU-device where the user should identify the wire for which the umbra shadow disappears. The second method is finding the umbra shadow disappearing wire with the optical densitometry measurements. For the computed radiography system, this can be done by the grey value histogram across the $\mathrm{Cd}$-wire shadows. Subsequently, knowing the diameter of the $\mathrm{Cd}$-wire and the distance between the wire and the imaging plate, one can calculate the effective collimation ratio. For the $\mathrm{OB}$ configuration the effective L/D was between 34.6 and 42.5 . For the $\mathrm{CB}$ configuration, on the other hand, these values increased to between 107 and 115, which is more than 100 (initial goal value). For both cases the values are less than geometric L/D ratios (46 for $\mathrm{OB}$ and 135 for $\mathrm{CB}$ ), which indicates to the existence of slight scattered neutron content.

\section{B. Beam Quality: Beam Purity Indicator (BPI) \& Sensitivity Indicator (SI)}

There are two indicators that are described in the ASTM E545 and can be used to define the designation of the quality level of a NR facility. The analysis of resulting radiographs of Beam Purity Indicator (BPI) and Sensitivity Indicator (SI) leads to determination of two important factors, which are the thermal neutron content and sensitivity level [5]. These two factors define the Category of the system by the ASTM designation of quality.

According to the ASTM E545 document, the visual analysis of the BPI shown in Fig. 7, two areas are of special interest: the cadmium wires (vertical lines) and the lead disks (lower two). If one of the $\mathrm{Cd}$ wires is significantly sharper than the other, which is the case for the $\mathrm{OB}$ configuration, the $\mathrm{L} / \mathrm{D}$ ratio of the system is considered to be low with the need to be improved. In contrast, for the $\mathrm{CB}$ configuration both $\mathrm{Cd}$ wires are comparably sharp. In addition to that, for the OB configuration, the lead disks (lower two) are distinguishable comparing to the polytetrafluoroethylene material of the BPI. That indicates to the high gamma content within the beam. Using the Bismuth single crystal in the $\mathrm{CB}$ configuration, we have filtered the beam from the gamma, which can be seen by the absence of lead disks in the BPI image.

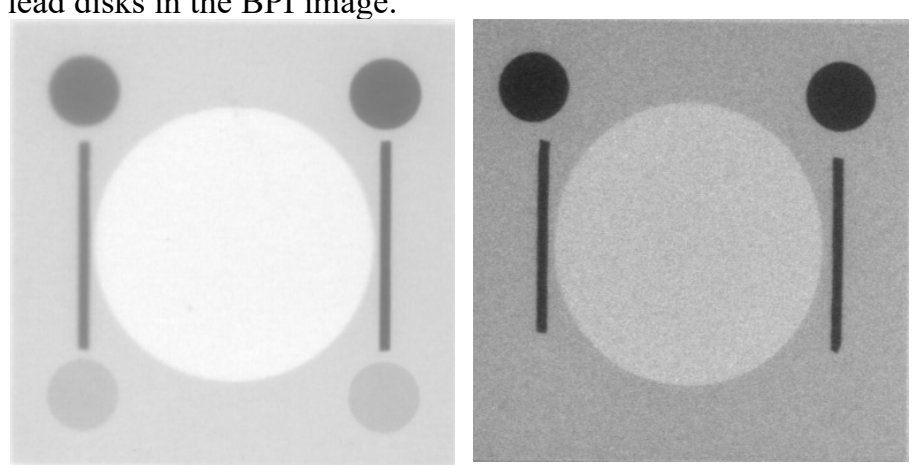

Fig. 8. The resulting image of the BPI for the OB (left) and CB (right) configurations. Note: the intensities of grey values are not comparable due to the lower neutron flux for the $\mathrm{CB}$ configuration.

The SI, on the other hand, is a step-wedge device containing gaps and holes of known dimensions that can be used to access the system based on the largest consecutive numbered visible hole $(\mathrm{H})$ and the thinnest visible gap $(\mathrm{G})$ between the thicknesschanging absorber steps. Basically, the analysis of SI radiograph can give the general idea on NR system's spatial resolution. 

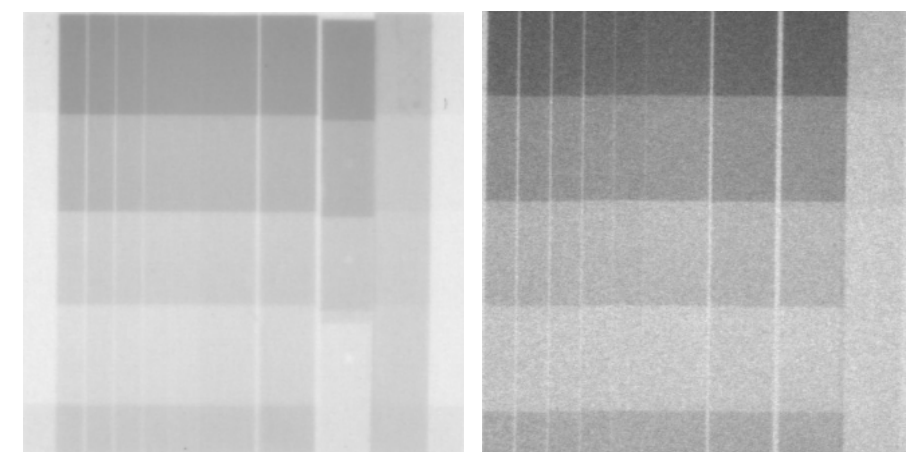

Fig. 9. The resulting image of the SI for the $\mathrm{OB}$ (left) and $\mathrm{CB}$ (right) configurations. Note: another SI has been used for the $\mathrm{CB}$ configuration that does not have the holes manufactured.

The optical densiometric analysis for different areas of the BPI and SI radiograph can be performed since the visual analysis can be somewhat challenging. To do so, the conversion of the gray values to the optical density values has been performed using the predicted calibration curve. The thermal neutron content (NC), as well as $\mathrm{H}$ and $\mathrm{G}$ values have been determined following the steps given in ASTM E545. For the $\mathrm{OB}$ configuration the $\mathrm{NC}$ was equal to 70.63 and the values for the $\mathrm{H}$ and $\mathrm{G}$ were equal to 4 and 6 , respectively. Those results let to the conclusion that the ASTM designation of quality level for the OB configuration corresponded to Category IV. For the $\mathrm{CB}$ configuration, on the other hand, the initial calculations showed the promising results of $\mathrm{NC}$ value being more than 75 and the $\mathrm{H}$ and $\mathrm{G}$ values both being 6 . Conventionally, more experiments including the film radiography are needed for the official and full declaration of the category; however, we are confident that all subsequent experiments will solidify the initial results that shows that the RSEC - NIF corresponds to the Category I facility by the ASTM designation of quality.

\section{CONCLUSIONS}

Although additional characterization experiments are needed, it is fair to say that after the installation of collimator components and the gamma filter, the new Neutron Imaging Facility at the Penn State Breazeale Nuclear Reactor (or RSEC) can produce images of high standards that corresponds to facility of Category I by ASTM designation of quality. The current system has an effective L/D ratio between 107 and 115 and can be increased to around 150 deviating the distance to the imaging plate and selecting different apertures with different diameters. The neutron flux measurement performed with gold foil activation technique at the exit surface of the beam port resulted in the average thermal neutron flux that is equal to $5.4 \times 10^{6} \mathrm{ncm}^{-2} \mathrm{~s}^{-1}$ for $1 \mathrm{MW}$ reactor powe $\mathrm{r}$ with the standard deviation of $6.4 \%$. Both mentioned values were in the scope of the initial objectives for the system and are successfully achieved. Further, the development of the neutron tomography capability is seen as one of the strategic next steps for the RSEC - NIF as well as the subsequent active application of our system in the field of NR.

\section{ACKNOWLEDGMENT}

The development process of the RSEC - NIF is financially supported by the internal funds of RSEC at Penn State. The authors would like to thank Alison Portanova, Jeffrey Geuther and other RSEC staff members for all their support.

\section{REFERENCES}

[1] K. Ünlü, B. J. Heidrich, "The Penn State Breazeale Reactor”, IAEA Technical Report Series No: 482History, Development and Future of TRIGA Research Reactors, IAEA Vienna, 2016.

[2] A. Kenges, K. Ünlü, J. Geuther, D. Beck, "Development of a New Neutron Imaging Facility for Radiation Science and Engineering at the Penn State University", Transactions of the American Nuclear Society, Vol. 123, November 2020, pp. 414-416 [Online]. Available: https://www.ans.org/pubs/transactions/article-48728/

[3] ASTM International, E 748: Standard Guide for Thermal Neutron Radiography of Materials, 1995

[4] ASTM International, E803: Standard Test Method for Determining the L/D Ratio of Neutron Radiography Beams, 2017

[5] ASTM International, E545: Standard Test Method for Determining Image Quality in Direct Thermal Neutron Radiographic Examination, 2014 